have implications on the development of assessment and management pathways for this under-recognised patient cohort.

\section{SINGLE-CELL RNA SEQUENCING REVEALS CARDIAC CELL-SPECIFIC TRANSCRIPTOMIC CHANGES IN DILATED CARDIOMYOPATHY}

${ }^{1} \mathrm{~A}$ Russell-Hallinan*, ${ }^{10}$ Cappa, ${ }^{1} \mathrm{~L}$ Kerrigan, ${ }^{2} \mathrm{C}$ Moravec, ${ }^{2} \mathrm{P}$ Collier, ${ }^{1} \mathrm{D}$ Simpson, ${ }^{1} \mathrm{C}$ Watson. 'Wellcome-Wolfson Institute for Experimental Medicine, Queens University Belfast, UK; ${ }^{2}$ Department of Cardiovascular and Metabolic Sciences, Lerner Research Institute, Cleveland Clinic, Ohio, USA

10.1136/heartjn|-2021-ICS.16

\section{NOT AVAILABLE FOR PUBLICATION}

\section{THE NATURAL HISTORY AND STAGING OF PRE-CLNICAL HEART FAILURE}

${ }^{1} \mathrm{~A}$ Moore, ${ }^{1} \mathrm{~A}$ Brennan, ${ }^{2} \mathrm{M}$ Ledwidge, ${ }^{1} \mathrm{~K}$ McDonald. ${ }^{1}$ St Vincent's University Hospital, Dublin, Ireland: ${ }^{2}$ Heartbeat Trust, Dublin, Ireland

\subsection{6/heartjnl-2021-ICS.17}

Introduction Heart failure (HF) is growing global epidemic. Given the high morbidity and mortality associated with HF, and the impact it has on an already stretched health care system, its effective management is a public health priority. Prevention is a critical component of this strategy and this is dependent on a more complete understanding of the natural history of the condition, especially its preclinical precursors.

Methods St Vincent's University Hospital offers a HF Prevention service (STOP-HF) targeted towards patients in its catchment area that have risk factors for HF. We retrospectively analysed the serial echocardiographic and natriuretic peptide data of 1,425 of these patients who have had at least two visits. Stage A was defined as no previous diagnosis of $\mathrm{HF}$ and a normal echocardiogram with one or more of the following: hypertension, hypercholesterolemia, obesity, vascular disease, diabetes mellitus, arrhythmia requiring therapy or significant valvular disease. Stage B, a cohort at higher risk of development of heart failure, was defined as no previous diagnosis of HF and evidence of a structural or functional abnormality on echocardiography including: ejection fraction $<50 \%$, diastolic dysfunction, left ventricular hypertrophy, valvular disease of at least moderate severity, an enlarged left ventricle or an abnormal LAVI. Demographics, comorbidities, medication use and biochemical data were also analysed from each visit.

Results A population of 1425 was analysed. On average, visit 2 was completed 4.8 years after visit 1 . The average age at visit 1 was $64 y$ rs with females accounting for $46 \%(n=649)$. At visit $1,72 \%(n=1022)$ of the population were classified as Stage A and $28 \%$ Stage B. The average BNP of a Stage A patient was $28 \mathrm{pg} / \mathrm{ml}$ while the average BNP of a Stage B patient was $64 \mathrm{pg} / \mathrm{ml}$. At visit 2, 60\% $(\mathrm{n}=858)$ of patients were classified as Stage A with $40 \%$ Stage B. At visit 2, the average BNP of a Stage A patient was $35 \mathrm{pg} / \mathrm{ml}$ while the average BNP of a Stage B patient was $141 \mathrm{pg} / \mathrm{ml}$. In terms of progression, $53 \%$ of patients remained in Stage A, 18\% progressed to Stage B, 21\% remained in Stage B and 7\% had regressed from Stage B to Stage A. The most prevalent manifestation of progression was an increase in LAVI while the notable change in patients demonstrating regression from Stage
B to Stage A was a reduction in LVH. In terms of changes in BNP between the two visits, those who remained in Stage A had an average BNP increase of $10 \mathrm{pg} / \mathrm{ml}$ from visit 1 to visit 2. Similarly, those who regressed from Stage B to Stage A had an average increase in BNP of $22 \mathrm{pg} / \mathrm{ml}$, whereas those that progressed from Stage A to Stage B had an average increase in BNP of $68 \mathrm{pg} / \mathrm{ml}$.

Summary This natural history study of a large sample of patients at risk for the development of heart failure demonstrates a significant proportion of patient with Stage B and furthermore a concerning progression rate of progression from Stage A to Stage B. Our data also identify a change in NP as a useful clinical biomarker of Stage B and risk of progression. It is hoped that this initial study will form as a baseline for further analysis and help guide screening and prevention strategies in the future

\section{MANAGEMENT AND OUTCOMES OF PATIENTS WITH LEFT ATRIAL APPENDAGE THROMBUS PRIOR TO PERCUTANEOUS CLOSURE. LAPTOP REGISTRY}

A McInerney, L Maroquin, G Tirado-Conte, P Salinas-Sanguino, L Nombela-Franco. Hospital Clínico San Carlos, Spain

10.1136/heartjnl-2021-ICS.18

NOT AVAILABLE FOR PUBLICATION

\section{YEAR RESULTS OF THE CARDIOLOGY TRAINEE FEEDBACK PROJECT}

N Fitzpatrick, BF McAdam, J Crowley, B McNeill, R Murphy, B Dalton. Irish Board for Training in Cardiovascular Medicine, Dublin, Ireland

\subsection{6/heartjnl-2021-ICS.19}

Background The Higher Specialist Training (HST) Scheme is run by the Royal College of Physicians (RCPI) overseen by the Irish Board for Training in Cardiovascular Medicine (IBTCM). The stated aim of the scheme is to provide doctors upon completion, with the necessary skills and knowledge to practice competently, professionally and independently as cardiology specialists. In order to inform dialogue surrounding improvements to the training scheme, those enrolled have been surveyed annually over the past 4 years to gage their views regarding the following domains - trainer/trainee interaction, access to training, procedural training, service commitments and training, research and audit, training program structure, gender balance, future career planning and location, quality of life and in the past year the impact of the Covid19 pandemic on training.

Methods For the past 4 years, the second author surveyed trainees in training years 1-4 through the medium of an online questionnaire. Trainees were asked to respond to a range of questions pertaining to the domains of interest listed above, predominantly indicating their level of agreement with a statement in the format of a 5 point Likert scale.

Results The response rate to the survey was excellent and averaged at $65 \%$ over the four years. Individual years ranged from $52 \%$ to a high of $82 \%$. Even in the year with the lowest response $(52 \%)$ a significant proportion of the trainees were represented. Overall trainees are happy with the training scheme with $88 \%$ saying they would recommend the 
training scheme. When asked if a trainee encountered significant challenges in accessing training - CT/MRI, followed by Adult Congenital Heart Disease and Electrophysiology were most frequently identified. Interest in dual accreditation was low, with $77 \%$ of respondents saying they did not intend to purse dual accreditation. Approximately half (47\%) of respondents felt that their year spent doing General Internal Medicine (GIM) enhanced their training experience. 46\% of respondents felt they have a poor or very poor work-life-balance, but $72 \%$ indicated that they had a very good or good quality of life. Future career planning: $83 \%$ of trainees indicated definitely or probably when asked if planning to work in Ireland after completion of training. However, when in 2018, a small number of trainees on overseas out of program experience were asked the same question a smaller proportion of respondents indicated that they wished to work in Ireland. In relation to gender - $28 \%$ of respondents were female, in keeping with prior work published by the authors in 2017 showing a 7:3 gender ratio overall for trainees between 1998 and 2017. To address the gender balance respondents felt that changes to the training scheme(63\%) and work practices (72\%) would be needed. Covid-19 had a marked affect on training, with $94 \%$ of trainees responding that it adversely affected their training.

Conclusion This 4 year study of the Irish cardiology trainees gives important insights into the strengths and weaknesses of the training scheme. It highlights the impact due to the current Covid-19 pandemic on training. It also gives valuable information regarding the future career goals of current trainees. All of the above, should be useful in the framing and shaping of any discussion regarding the future of cardiology training and service delivery in Ireland in the decades to come.

\section{BENEFITS OF 3D PRINTING IN PLANNING AND COMMUNICATION IN ADULT CARDIAC SURGERY: A PILOT STUDY}

${ }^{1} \mathrm{G}$ Beattie, ${ }^{2} \mathrm{~A}$ Graham. ${ }^{1}$ Royal Victoria Hospital, Belfast, UK; ${ }^{2}$ Belfast Trust, Belfast UK

\subsection{6/heartjn|-2021-ICS.20}

Introduction 3D printing is now cost effective and, with specialist companies handling the complexity of making models, it is now relatively easy to get an accurate surgical model printed. Simulation surgery is now becoming a reality to highlight areas of concern in an operation. Some orthopaedic surgeons are using models to practice the operation before ever laying a hand on a patient, so the authors wanted to investigate if $3 \mathrm{D}$ printed models would help with planning and communication in adult cardiac surgery.

Methods A local company who prints 3D medical models was given a stimulus grant to from June 2020 to March 2021. They were able to offer models for free to local specialist surgery departments. When the surgery was complete an online assessment form was completed to review the helpfulness of the models, and all of these forms were collated from the company for the cardiac models ordered during the study period. The data was analysed in Microsoft Excel. CT scans were sent electronically to the company. They created the model using proprietary software and an interactive model was sent to the surgeon using sketchfab. This was viewed by the requesting surgeon who made changes as necessary. When

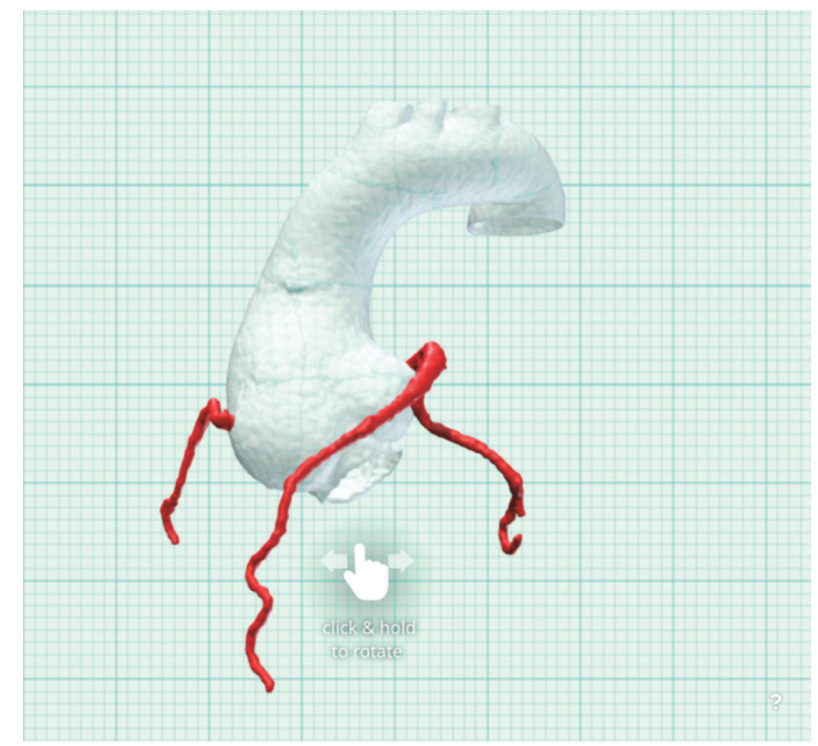

Abstract 20 Figure 1 Image of the model as it appears to the surgeon. In the software it is interactive so the surgeon can rotate and if the model is sliced this can be manipulated to allow viewing of the internal structure of the heart model

Link to interactive image: https://sketchfab.com/3d-models/p03018db0590ae83954351877edcc38776e6a2

the model was approved it was then printed. Different materials could be requested differing in flexibility to opacity. The company used Formlabs 2 and Formlabs 3B printers.

Results Sixteen 3D Models were made to aid planning of adult cardiac surgery, 6 for valve surgery, 4 for aortic root surgery, 4 for CABG surgery, 1 for a myxoma and 1 case for post-infarction VSD. In 9 cases (56\%), the surgeon changed their plan for the surgery after viewing the model and in 2 cases $(12 \%)$ it changed the diagnosis. In 15 cases (94\%) the model was felt to have a positive effect on patient safety and in all cases it was felt to be helpful for communication with other surgeons and trainees.

Conclusion Our pilot study suggests that 3D printed models have the potential to improve safety and communication in certain types of adult cardiac surgery and merit further study. Link to interactive image https://sketchfab.com/3d-models/ p03018-db0590ae83954351877edcc38776e6a2.

\section{SURGICAL AORTIC VALVE REPLACEMENT: EARLIER VS NEWER GENERATION BIOPROSTHETIC VALVES - A COMPARISON OF EARLY HEMODYNAMIC PERFORMANCE}

${ }^{1,2} \mathrm{G}$ Keehan, ${ }^{1} \mathrm{~A}$ Vainorius, ${ }^{1} \mathrm{~A}$ White, ${ }^{1} \mathrm{~A}$ Soo. ${ }^{1}$ Department of Cardiothoracics, University Hospital Galway, Ireland; ${ }^{2}$ National University of Ireland, Galway

\subsection{6/heartjnl-2021-ICS.21}

Objectives Modern advances in bioprosthetic valve tissue technology have resulted in the development of newer generation bioprosthetic aortic valves such as the Edwards Lifesciences ${ }^{\circledR}$ Inspiris Resilia ${ }^{\mathrm{TM}}$ (Inspiris) and the Medtronic ${ }^{\circledR}$ Avalus $^{\mathrm{TM}}$ Bioprosthesis (Avalus).

These claim to exhibit improved hemodynamic sustainability, and prevention from structural valve deterioration, translating into long-term durability; in comparison to bioprosthetic 